\title{
On the Complexity of Closest Pair via Polar-Pair of Point-Sets
}

\author{
Roee David ${ }^{1}$ \\ Datorama \\ Israel \\ daroeevid@gmail.com
}

Karthik C. S. ${ }^{2}$

Weizmann Institute of Science

Rehovot, Israel

karthik.srikanta@weizmann.ac.il

Bundit Laekhanukit ${ }^{3}$

Shanghai University of Finance and Economics

Shanghai, China

Max-Planck-Institute for Informatics

Saabrücken, Germany

blaekhan@mpi-inf.mpg.de

\begin{abstract}
Every graph $G$ can be represented by a collection of equi-radii spheres in a $d$-dimensional metric $\Delta$ such that there is an edge $u v$ in $G$ if and only if the spheres corresponding to $u$ and $v$ intersect. The smallest integer $d$ such that $G$ can be represented by a collection of spheres (all of the same radius) in $\Delta$ is called the sphericity of $G$, and if the collection of spheres are non-overlapping, then the value $d$ is called the contact-dimension of $G$. In this paper, we study the sphericity and contact dimension of the complete bipartite graph $K_{n, n}$ in various $L^{p}$-metrics and consequently connect the complexity of the monochromatic closest pair and bichromatic closest pair problems.
\end{abstract}

2012 ACM Subject Classification Theory of computation $\rightarrow$ Computational geometry

Keywords and phrases Contact dimension, Sphericity, Closest Pair, Fine-Grained Complexity

Digital Object Identifier 10.4230/LIPIcs.SoCG.2018.28

Related Version A full version of this paper is available at http://arxiv.org/abs/1608.03245

Acknowledgements We would like to thank Aviad Rubinstein for sharing with us [26] and for the many discussions. We would like to thank Petteri Kaski and Rasmus Pagh for useful discussions and also for pointing out the reference [3]. We would like to thank Eylon Yogev and Amey Bhangale for some preliminary discussions. We would like to thank Uriel Feige for a lot of useful comments and discussions. Finally, we would like to thank Roei Tell for helping us improving the presentation of the paper.

1 This work was partially supported by the ISF grant \# 621/12) and by the I-CORE program grant \# $4 / 11)$.

2 This work was partially supported by Irit Dinur's ERC-StG grant \# 239985 and ERC-CoG grant \# 772839 .

3 This work was partially supported by the ISF grant \# 621/12 and by the I-CORE program grant \# 4/11). Part of this work was done while the third author was visiting the Simons Institute for the Theory of Computing. It was partially supported by the DIMACS/Simons Collaboration on Bridging Continuous and Discrete Optimization through the NSF grant \# CCF-1740425.

(c) (i) () Roee David, Karthik C. S. and Bundit Laekhanukit;

34th International Symposium on Computational Geometry (SoCG 2018).

Editors: Bettina Speckmann and Csaba D. Tóth; Article No. 28; pp. 28:1-28:15

Leibniz International Proceedings in Informatics 


\section{Introduction}

This paper studies the geometric representation of a complete bipartite graph in $L^{p}$-metrics and consequently connects the complexity of the closest pair and bichromatic closest pair problems beyond certain dimensions. Given a point-set $P$ in a $d$-dimensional $L^{p}$-metric, an $\alpha$-distance graph is a graph $G=(V, E)$ with a vertex set $V=P$ and an edge set

$$
E=\left\{u v:\|u-v\|_{p} \leq \alpha ; u, v \in P ; u \neq v\right\}
$$

In other words, points in $P$ are centers of spheres of radius $\alpha / 2$, and $G$ has an edge $u v$ if and only if the spheres centered at $u$ and $v$ intersect. The sphericity of a graph $G$ in an $L^{p}$-metric, denoted by $\operatorname{sph}_{p}(G)$, is the smallest dimension $d$ such that $G$ is isomorphic to some $\alpha$-distance graph in a $d$-dimensional $L^{p}$-metric, for some constant $\alpha>0$. The sphericity of a graph in the $L^{\infty}$-metric is known as cubicity. A notion closely related to sphericity is contact-dimension, which is defined in the same manner except that the spheres representing $G$ must be non-overlapping. To be precise, an $\alpha$-contact graph $G=(V, E)$ of a point-set $P$ is an $\alpha$-distance graph of $P$ such that every edge $u v$ of $G$ has the same distance (i.e., $\left.\|u-v\|_{p}=\alpha\right)$. Thus, $G$ has the vertex set $V=P$ and has an edge set $E$ such that

$\forall u v \in E, \quad\|u-v\|_{p}=\alpha \quad$ and $\quad \forall u v \notin E, \quad\|u-v\|_{p}>\alpha$.

The contact-dimension of a graph $G$ in the $L^{p}$-metric, denoted by $\mathrm{cd}_{p}(G)$, is the smallest integer $d \geq 1$ such that $G$ is isomorphic to a contact-graph in the $d$-dimensional $L^{p}$-metric. We will use distance and contact graphs to means 1-distance and 1-contact graphs.

We are interested in determining the sphericity and the contact-dimension of the biclique $K_{n, n}$ in various $L^{p}$-metrics. For notational convenience, we denote $\operatorname{sph}_{p}\left(K_{n, n}\right)$ by bsph $\left(L^{p}\right)$, the biclique sphericity of the $L^{p}$-metric, and denote $\mathrm{cd}_{p}\left(K_{n, n}\right)$ by $\operatorname{bcd}\left(L^{p}\right)$, the biclique contact-dimension of the $L^{p}$-metric. We call a pair of point-sets $(A, B)$ polar if it is the partition of the vertex set of a contact graph isomorphic to $K_{n, n}$. More precisely, a pair of point-sets $(A, B)$ is polar in an $L^{p}$-metric if there exists a constant $\alpha>0$ such that every inner-pair $u, u^{\prime} \in A$ (resp., $v, v^{\prime} \in B$ ) has $L^{p}$-distance greater than $\alpha$ while every crossing-pair $u \in A, v \in B$ has $L^{p}$-distance exactly $\alpha$.

The biclique sphericity and contact-dimension of the $L^{2}$ and $L^{\infty}$ metrics are well-studied in literature (see $[25,21,22,11,23,9]$ ). Maehara $[23,21]$ showed that $n<\operatorname{bsph}\left(L^{2}\right) \leq(1.5) n$, and Maehara and Frankl \& Maehara $[22,11]$ showed that $(1.286) n-1<\operatorname{bcd}\left(L^{2}\right)<(1.5) n$. For cubicity, Roberts [25] showed that $\operatorname{bcd}\left(L^{\infty}\right)=\operatorname{bsph}\left(L^{\infty}\right)=2 \log _{2} n$. Nevertheless, for other $L^{p}$-metrics, contact dimension and sphericity are not well-studied.

\subsection{Our results and contributions}

Our main conceptual contribution is connecting the complexity of the (monochromatic) closest pair problem (CLOSEST PAIR) to that of the bichromatic closest pair problem (BCP) through the contact dimension of the biclique. This is discussed in subsection 1.1.1. Our main technical contributions are bounds on the contact dimension and sphericity of the biclique for various $L^{p}$-metrics. This is discussed in subsection 1.1.2. Finally, as an application of the connection discussed in subsection 1.1.1 and the bounds discussed in subsection 1.1.2, we show computational equivalence between monochromatic and bichromatic closest pair problems. 


\subsubsection{Connection between Closest Pair and BCP}

In Closest PAIR, we are asked to find a pair of points in a set of $m$ points with minimum distance. BCP is a generalization of CLOSEST PAIR, in which each point is colored red or blue, and we are asked to find a pair of red-blue points (i.e., bichromatic pair) with minimum distance. It is not hard to see that BCP is at least as hard as CLOSEST PAIR since we can apply an algorithm for BCP to solve CLOSEST PAIR with the same asymptotic running time. However, it is not clear whether the other direction is true. We will give a simple reduction from BCP to Closest PAIR using a polar-pair of point-sets. First, take a polar-pair $(A, B)$, each with cardinality $n=m / 2$, in the $L^{p}$-metric. Next, pair up vectors in $A$ and $B$ to red and blue points, respectively, and then attach a vertex $u \in A$ (resp., $v \in B$ ) to its matching red (resp., blue) point. This reduction increases the distances between every pair of points, but by the definition of the polar-pair, this process has more effect on the distances of the monochromatic (i.e., red-red or blue-blue) pairs than that of bichromatic pairs, and the reduction, in fact, has no effect on the order of crossing-pair distances at all. By scaling the vectors in $A$ and $B$ appropriately, this gives an instance of CLOSEST PAIR whose closest pair of points is bichromatic. Consequently, provided that the polar-pair of point-sets $(A, B)$ in a $d$-dimensional metric can be constructed within a running time at least as fast as the time for computing CLOSEST PAIR in the same metric, this gives a reduction from BCP to Closest PAIR, thus implying that they have the same running time lower bound.

\subsubsection{Bounds on contact dimension and sphericity of biclique}

Our main technical results are lower and upper bounds on the biclique contact-dimension for the $L^{p}$-metric space where $p \in \mathbb{R}_{\geq 1} \cup\{0\}$.

- Theorem 1. The following are upper and lower bounds on biclique contact-dimension for the $L^{p}$-metric.

$$
\begin{array}{cr}
\operatorname{bsph}\left(L^{0}\right)=\operatorname{bcd}\left(L^{0}\right)=n & \\
\left.n \leq \operatorname{bsph}\left(L_{\{0,1\}}^{0}\right)\right) \leq \operatorname{bcd}\left(L_{\{0,1\}}^{0}\right) \leq n^{2} & \left(\text { i.e., } P \subseteq\{0,1\}^{d}\right) \\
\Omega(\log n) \leq \operatorname{bsph}\left(L^{1}\right) \leq \operatorname{bcd}\left(L^{1}\right) \leq n^{2} & \\
\Omega(\log n) \leq \operatorname{bsph}\left(L^{p}\right) \leq \operatorname{bcd}\left(L^{p}\right) \leq 2 n & \text { for } p \in(1,2) \\
\operatorname{bsph}\left(L^{p}\right)=\Theta\left(\operatorname{bcd}\left(L^{p}\right)\right)=\Theta(\log n) & \text { for } p>2
\end{array}
$$

Note that $\operatorname{bsph}(\Delta) \leq \operatorname{bcd}(\Delta)$ for any metric $\Delta$. Thus, it suffices to prove a lower bound for $\operatorname{bsph}(\Delta)$ and prove an upper bound for $\operatorname{bcd}(\Delta)$.

We note that the bounds on the sphericity and the contact dimension of the $L^{1}$-metric in (3) are obtained from (5) and (1), respectively. We are unable to show a strong (e.g., linear) lower bound for the $L^{1}$-metric. However, we prove the weaker (average-case) result below for the $L^{1}$-metric which can be seen as a progress toward proving stronger lower bounds on the sphericity of the biclique in this metric (see Corollary 7 for more discussion on its applications).

- Theorem 2. For any integer $d>0$, there exist no two finite-supported random variables $X, Y$ taking values from $\mathbb{R}^{d}$ such that the following hold.

$$
\begin{gathered}
\underset{x_{1}, x_{2} \in_{R} X}{\mathbb{E}}\left[\left\|x_{1}-x_{2}\right\|_{1}\right]>\underset{x_{1} \in \underset{R}{X} X, y_{1} \in_{R} Y}{\mathbb{E}}\left[\left\|x_{1}-y_{1}\right\|_{1}\right] \\
\underset{y_{1}, y_{2} \in_{R} Y}{\mathbb{E}}\left[\left\|y_{1}-y_{2}\right\|_{1}\right]>\underset{x_{1} \in \underset{R}{X} X, y_{1} \in_{R} Y}{\mathbb{E}}\left[\left\|x_{1}-y_{1}\right\|_{1}\right] .
\end{gathered}
$$


Table 1 Known bounds on sphericity and contact dimension of biclique.

\begin{tabular}{|l||l|l|}
\hline \multicolumn{1}{|c|}{ Metric } & \multicolumn{1}{|c|}{ Bound } & \multicolumn{1}{c|}{ From } \\
\hline$L^{0}$ & $\operatorname{bsph}\left(L^{0}\right)=\operatorname{bcd}\left(L^{0}\right)=n$ & This paper \\
$L^{1}$ & $\Omega(\log n) \leq \mathrm{bsph}\left(L^{1}\right) \leq \mathrm{bcd}\left(L^{1}\right) \leq n^{2}$ & This paper \\
$L^{p}, p \in(1,2)$ & $\Omega(\log n) \leq \mathrm{bsph}\left(L^{p}\right) \leq \mathrm{bcd}\left(L^{p}\right) \leq 2 n$ & This paper \\
$L^{2}$ & $n<\mathrm{bsph}\left(L^{2}\right) \leq \mathrm{bcd}\left(L^{2}\right)<1.5 \cdot n$ & {$[23,11]$} \\
$L^{p}, p>2$ & $\operatorname{bsph}\left(L^{p}\right)=\Theta\left(\mathrm{bcd}\left(L^{p}\right)\right)=\Theta(\log n)$ & This paper \\
$L^{\infty}$ & $\operatorname{bsph}\left(L^{\infty}\right)=\operatorname{bcd}\left(L^{\infty}\right)=2 \log _{2} n$ & {$[25]$} \\
\hline
\end{tabular}

For an overview on the known bounds on bsph and bcd (including the results in this paper), please see Table 1 .

In the full version of the paper, we give an alternate proof of the linear lower bound on $\operatorname{bsph}\left(L^{2}\right)$ using spectral analysis similar to that in [9]. While our lower bound is slightly weaker than the best known bounds [11,23], our arguments require no heavy machinery and thus are arguably simpler than the previous works $[11,23,9]$.

Alman and Williams [3] showed the subquadratic-time hardness for BCP in $L^{p}$-metrics, for all $p \in \mathbb{R}_{\geq 1} \cup\{0\}$, under the Orthogonal Vector Hypothesis (OVH). From Theorem 1 and the connection between BCP and CLOSEST PAIR described in subsection 1.1.1, we have the following hardness of CLOSEST PAIR.

- Theorem 3. Let $p>2$. For any $\varepsilon>0$ and $d=\omega(\log n)$, the closest pair problem in the $d$-dimensional $L^{p}$-metric admits no $\left(n^{2-\varepsilon}\right)$-time algorithm unless the Orthogonal Vectors Hypothesis is false.

We remark here that showing conditional hardness for CLOSEST PAIR in the $L^{p}$ metric for $p \leq 2$ remains an outstanding open problem ${ }^{4}$. Recently, Rubinstein [26] showed that the subquadratic-time hardness holds even for approximating BCP: Assuming OVH, for every $p \in \mathbb{R}_{\geq 1} \cup\{0\}$ and every $\varepsilon>0$, there is a constant $\gamma(\varepsilon, p)>0$ such that there is no $(1+\gamma)$-approximation algorithm running in time $O\left(n^{2-\varepsilon}\right)$ for BCP in the $L^{p}$-metric. By using the connection between BCP and ClOSEST PAIR described in subsection 1.1.1 and the bounds in Theorem 1 (to be precise we need the efficient construction with appropriate gap as given by Theorem 17), the hardness of approximation result can be extended to Closest Pair for $L^{p}$ metrics where $p>2$.

- Theorem 4. Let $p>2$. For every $\varepsilon>0$ and $d=\omega(\log n)$, there exists a constant $\gamma=\gamma(p, \varepsilon)>0$ such that the closest pair problem in the d-dimensional $L^{p}$-metric admits no $\left(n^{2-\varepsilon}\right)$-time $(1+\gamma)$-approximation algorithm unless the Orthogonal Vectors Hypothesis is false.

We remark that the hardness for the case of the $L^{\infty}$-metric does not follow (at least directly) from [3] or [26]. For independent interest, we show the subquadratic-time hardness of BCP and Closest PAIR in the $L^{\infty}$-metric.

- Theorem 5. For any $\varepsilon>0$ and $d=\omega(\log n)$, the closest pair problem in the $d$-dimensional $L^{\infty}$-metric admits no $\left(n^{2-\varepsilon}\right)$-time $(2-o(1))$-approximation algorithm unless the Orthogonal Vectors Hypothesis is false.

4 The subquadratic-time hardness of Closest PAIR in the $L^{p}$-metric for $p \in \mathbb{R}_{\geq 1} \cup\{0\}$ was claimed in [1] but later retracted [2]. 
We note that the lower bounds on bsph act as barriers for gadget reductions from BCP to Closest Pair. This partially explains why there has been no progress in showing conditional hardness for CLOSEsT PAIR in the Euclidean metric for $d=\omega(\log n)$ dimensions (as bsph $\left(L^{2}\right)=\Omega(n)$ ). In addition, Rubinstein noted in [26] that one obstacle in proving inapproximability results for CLOSEST PAIR is due to the triangle inequality - any two pointsets $A$ and $B$ in any metric space cannot have distinct points $a, a^{\prime} \in A$ and $b \in B$ such that $\left\|a-a^{\prime}\right\|>2 \cdot \max \left\{\|a-b\|,\left\|a^{\prime}-b\right\|\right\}$ (as it would violate the triangle inequality). This rules out the possibility of obtaining the conditional hardness for 2-approximating CLOSEST PAIR for any metric via simple gadget reductions. We note that the inapproximability factor of Theorem 5 matches the triangle inequality barrier (for the $L^{\infty}$ metric).

\subsection{Related works}

While our paper studies sphericity and contact-dimension of the complete bipartite graph, determining the contact-dimension of a complete graph in $L^{p}$-metrics has also been extensively studied in the notion of equilateral dimension. To be precise, the equilateral dimension of a metric $\Delta$ which is the maximum number of equidistant points that can be packed in $\Delta$. An interesting connection is in the case of the $L^{1}$-metric, for which we are unable to establish a strong lower bound for $\operatorname{bsph}\left(L^{1}\right)$. The equilateral dimension of $L^{1}$ is known to be at least $2 d$, and this bound is believed to be tight [14]. This is a notorious open problem known as Kusner's conjecture, which is confirmed for $d=2,3,4[5,20]$, and the best upper bound for $d \geq 5$ is $O(d \log d)$ by Alon and Pavel [4]. If Kusner's conjecture is true for all $d$, then $\operatorname{sph}_{1}\left(K_{n}\right)=n / 2$.

The complexity of Closest PAIR has been a subject of study for many decades. There have been a series of developments on Closest PAIR in the Euclidean space (see, e.g., $[7,15,19,27,8])$, which culminates in a deterministic $O\left(2^{O(d)} n \log n\right)$-time algorithm [8] and a randomized $O\left(2^{O(d)} n\right)$-time algorithm $[24,19]$. For low (i.e., constant) dimensions, these algorithms are tight as the matching lower bound of $\Omega(n \log n)$ was shown by Ben-Or [6] and Yao [33] for the algebraic decision tree model, thus settling the complexity of CLOSEST PAIR in low dimensions. For high dimensions (i.e., $d=\omega(\log n))$, there is no known algorithm that runs in time significantly better than a trivial $O\left(n^{2} d\right)$-time algorithm for general $d$ except for the case that $d \geq \Omega(n)$ whereas there are subcubic-time algorithms in $L^{1}$ and $L^{\infty}$ metrics $[12,16]$.

In the last few years, there has been a lot of progress in our understanding of $\mathrm{BCP}$, Closest PAIR, and related problems. Alman and Williams [3] showed subquadratic time hardness for $\mathrm{BCP}$ in $d=\omega(\log n)$ dimensions under $\mathrm{OVH}$ in the $L^{p}$ metric for every $p \in \mathbb{R}_{\geq 1} \cup\{0\}$. Williams [30] extended the result of [3] and showed the above subquadratictime hardness for BCP even for dimensions $d=\omega\left((\log \log n)^{2}\right)$ under OVH. In a recent breakthrough on hardness of approximation in P, Abboud et al. [2] showed the subquadratictime hardness for approximating the Bichromatic Maximum Inner Product problem under OVH in the $L^{p}$ metric for every $p \in \mathbb{R}_{\geq 1} \cup\{0\}$, and the result holds for almost polynomial approximation factors. More recently, building upon the ideas in [2], Rubinstein [26] showed under OVH the inapproximablility of BCP for every $L^{p}$-metric for $p \in \mathbb{R}_{\geq 1} \cup\{0\}$.

\section{Preliminaries}

We use the following standard terminologies and notations. 
Distance measures. For any vector $x$ in $\mathbb{R}^{d}$, we denote by $\|x\|_{p}$ the $L^{p}$-norm of $x$ and is equal to $\left(\sum_{i=1}^{d}\left|x_{i}\right|^{p}\right)^{1 / p}$. The $L^{\infty}$-norm of $x$ is denoted by $\|x\|_{\infty}=\max _{i \in[d]}\left\{\left|x_{i}\right|\right\}$, and the $L^{0}$-norm of $x$ is denoted by $\|x\|_{0}=\left|\left\{x_{i} \neq 0: i \in[d]\right\}\right|$, i.e., the number of non-zero coordinates of $x$. These norms define distance measures in $\mathbb{R}^{d}$. The distance of two points $x$ and $y$ w.r.t. the $L^{p}$-norm, say $L^{p}$-distance, is thus $\|x-y\|_{p}$. The distance measures that are well studied in literature are the Hamming distance $L^{0}$-norm, the Rectilinear distance $L^{1}$-norm, the Euclidean distance $L^{2}$-norm, the Chebyshev distance (a.k.a, Maximum-norm) $L^{\infty}$-norm.

Problems. Here we give formal definitions of Closest Pair and BCP. In Closest Pair, we are given a collection of points $P \subseteq \mathbb{R}^{d}$ in a $d$-dimensional $L^{p}$-metric, and the goal is find a pair of distinct points $a, b \in P$ that minimizes $\|u-v\|_{p}$. In $\mathrm{BCP}$, the input point-set is partitioned into two color classes (the collections of red and blue points) $A$ and $B$, and the goal is find a pair of points $u \in A$ and $v \in B$ that minimizes $\|u-v\|_{p}$.

Fine-grained complexity and conditional hardness. Conditional hardness is the current trend in proving running-time lower bounds for polynomial-time solvable problems. This has now developed into the area of Fine-Grained Complexity. Please see, e.g., [31, 32] and references therein.

The Orthogonal Vectors Hypothesis (OVH) is a popular complexity theoretic assumption in Fine-Grained Complexity. OVH states that in the Word RAM model with $O(\log n)$ bit words, any algorithm requires $n^{2-o(1)}$ time in expectation to determine whether collections of vectors $A, B \subseteq\{0,1\}^{d}$ with $|A|=|B|=n / 2$ and $d=\omega(\log n)$ contain an orthogonal pair $u \in A$ and $v \in B$ (i.e., $\left.\sum_{i=1}^{d} u_{i} \cdot v_{i}=0\right)$.

Another popular conjecture is the Strong Exponential-Time Hypothesis for SAT (SETH), which states that, for every $\varepsilon>0$, there exists an integer $k_{\varepsilon}$ such that $k_{\varepsilon}$-SAT on $n$ variables cannot be solved in $O\left(2^{(1-\varepsilon) n}\right)$-time. Williams showed that SETH implies OVH [29].

\section{$3 \quad$ Representing biclique in $L^{1}$}

In this section, we discuss the case of the $L^{1}$-metric. As discussed in the introduction, this is the only case where we are unable to prove neither strong lower bound nor linear upper bound. A weak lower bound $\operatorname{bsph}\left(L^{1}\right) \geq \Omega(\log n)$ follows from the proof for the $L^{p}$-metric with $p>2$ in Section 6.1 (Theorem 16), and a quadratic upper bound $\operatorname{bcd}\left(L^{1}\right) \leq n^{2}$ follows from the proof for the $L^{0}$-metric in Section 4.2 (Corollary 12). However, we cannot prove any upper bound smaller than $\Omega\left(n^{2}\right)$ or any lower bound larger than $O(\log n)$. Hence, we study an average case relaxation of the question.

We show in Theorem 2 that there is no distribution whose expected distances simulate a polar-pair of point-sets in the $L^{1}$-metric. Consequently, even though we could not prove the biclique sphericity lower bound for the $L^{1}$-metric, we are able to refute an existence of a geometric representation with large gap for any dimension as shown in Corollary 7 . (A similar result was shown in [10] for the $L^{2}$-metric.)

- Definition 6 ( $L^{1}$-distribution). For any $d>0$, let $X, Y$ be two random variables taking values from $\mathbb{R}^{d}$. An $L^{1}$-distribution is constructed by $X, Y$ if the following holds.

$$
\begin{gathered}
\underset{x_{1}, x_{2} \in R}{\mathbb{E}}\left[\left\|x_{1}-x_{2}\right\|_{1}\right]>\underset{x_{1} \in \underset{R}{X} X, y_{1} \in_{R} Y}{\mathbb{E}}\left[\left\|x_{1}-y_{1}\right\|_{1}\right], \\
\underset{y_{1}, y_{2} \in_{R} Y}{\mathbb{E}}\left[\left\|y_{1}-y_{2}\right\|_{1}\right]>\underset{x_{1} \in \in_{R} X, y_{1} \in_{R} Y}{\mathbb{E}}\left[\left\|x_{1}-y_{1}\right\|_{1}\right] .
\end{gathered}
$$


- Theorem 2 (Restated). For any two finite-supported random variables $X, Y$ that are taking values from $\mathbb{R}^{d}$, there is no $L^{1}$-distribution.

Proof. Assume towards a contradiction that there exist two finite-supported random variables $X, Y$ that are taking values in $\mathbb{R}^{d}$ and that are satisfying Eq. 6 of Definition 6 . Given a vector $x \in \mathbb{R}^{d}$, we denote by $x(i)$ the value of the $i$-th coordinate of $x$. Hence the following inequalities hold,

$$
\begin{aligned}
& 0>\underset{x_{1} \in_{R} X, y_{1} \in_{R} Y}{\mathbb{E}}\left[\left\|x_{1}-y_{1}\right\|_{1}\right]-\underset{x_{1}, x_{2} \in_{R} X}{\mathbb{E}}\left[\left\|x_{1}-x_{2}\right\|_{1}\right] \\
& =\underset{x_{1}, x_{2} \in_{R} X, y_{1} \in_{R} Y}{\mathbb{E}}\left[\left\|x_{1}-y_{1}\right\|_{1}-\left\|x_{1}-x_{2}\right\|_{1}\right] \\
& =\frac{1}{d} \cdot \underset{x_{1}, x_{2} \in R}{\mathbb{E} X, y_{1} \in_{R} Y}\left[\underset{i \in_{R}[1 \ldots d]}{\mathbb{E}}\left[\left|x_{1}(i)-y_{1}(i)\right|-\left|x_{1}(i)-x_{2}(i)\right|\right]\right] \\
& =\frac{1}{d} \cdot \underset{i \in_{R}[1 \ldots d]}{\mathbb{E}}\left[\underset{x_{1}, x_{2} \in_{R} X, y_{1} \in_{R} Y}{\mathbb{E}}\left[\left|x_{1}(i)-y_{1}(i)\right|-\left|x_{1}(i)-x_{2}(i)\right|\right]\right] \text {. }
\end{aligned}
$$

Thus for some $i^{\star} \in[d]$ the following holds,

$$
0>\underset{x_{1}, x_{2} \in R}{\mathbb{E} X, y_{1} \in_{R} Y}\left[\left|x_{1}\left(i^{\star}\right)-y_{1}\left(i^{\star}\right)\right|-\left|x_{1}\left(i^{\star}\right)-x_{2}\left(i^{\star}\right)\right|\right] .
$$

Fix $i^{\star} \in[d]$ satisfying the above inequality. For the sake of clarity, we assume that the random variables $X, Y$ are taking values in $\mathbb{R}$ (i.e., projection on the $i^{\star \text { th }}$ coordinate). We can assume that the size of $\operatorname{supp}(X) \cup \operatorname{supp}(Y)$ is greater than 1 because, if $\operatorname{supp}(X) \cup$

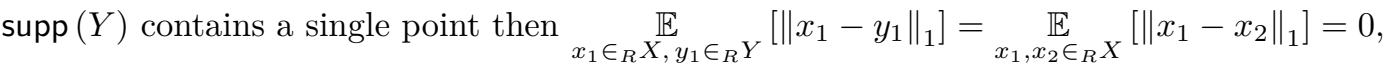
contradicting Eq. 7. Let $\operatorname{supp}(X) \cup \operatorname{supp}(Y)$ contains $t \geq 2$ points. We prove by induction on $t$, that there are no $X, Y$ over $\mathbb{R}$ satisfying Eq. 7 . The base case is when $t=2$. By Eq. 7 , there exists 3 points $\tilde{x}_{1}, \tilde{x}_{2}, \tilde{y}_{1}$ in $\mathbb{R}$ such that,

$$
0>\left\|\tilde{x}_{1}-\tilde{y}_{1}\right\|_{1}-\left\|\tilde{x}_{1}-\tilde{x}_{2}\right\|_{1} .
$$

Since supp $(X) \cup \operatorname{supp}(Y)$ contains exactly two points, $\tilde{x}_{1}, \tilde{x}_{2}, \tilde{y}_{1}$ are supported by two distinct points in $\mathbb{R}$. Hence, there are two cases, either that $x_{1}=x_{2}$ (and $y_{1} \neq x_{1}$ ) or that $x_{1} \neq x_{2}$ (and either $\tilde{y}_{1}=\tilde{x}_{1}$ or $\tilde{y}_{1}=\tilde{x}_{2}$ ). It is easy to see that none of these cases satisfy Eq. 8, a contradiction.

Assume the induction hypothesis that there are no $X, Y$ taking values from $\mathbb{R}$ satisfying Eq. 7 when the size of $\operatorname{supp}(X) \cup \operatorname{supp}(Y)$ is equal to $k \geq 2$. Then consider the case when $t=k+1 \geq 3$. Sort the points in $\operatorname{supp}(X) \cup \operatorname{supp}(Y)$ by their values, and denote by $s_{i}$ the value of the $i$-th point of $\operatorname{supp}(X) \cup \operatorname{supp}(Y)$. For the sake of simplicity, we say that we change the value of $s_{t-1}$ to $\tilde{s}_{t-1}$, where $s_{t-2} \leq \tilde{s}_{t-1} \leq s_{t}$, if after changing its value we change the values of (at least one of) $X, Y$ to $\tilde{X}, \tilde{Y}$ in such a way that the value of the $(t-1)$-th point (after sorting) of $\operatorname{supp}(\tilde{X}) \cup \operatorname{supp}(\tilde{Y})$ is equal to $\tilde{s}_{t-1}$ (if $s_{t-2}=\tilde{s}_{t-1}$, then the value of the $(t-2)$-th point of $\operatorname{supp}(\tilde{X}) \cup \operatorname{supp}(\tilde{Y})$ is equal to $\left.\tilde{s}_{t-1}\right)$. Define the function $f:\left[s_{t-2}, s_{t}\right] \rightarrow \mathbb{R}$ as follows:

$$
f(x)=\underset{x_{1} \in R}{\mathbb{X}, y_{1} \in_{R} \tilde{Y}}\left[\left\|x_{1}-y_{1}\right\|_{1}\right]-\underset{x_{1}, x_{2} \in_{R} \tilde{X}}{\mathbb{E}}\left[\left\|x_{1}-x_{2}\right\|_{1}\right],
$$

where $\tilde{X}, \tilde{Y}$ are obtained after changing $s_{t-1}$ to $x \in\left[s_{t-2}, s_{t}\right]$. The crucial observation is that the function $f$ is linear. Hence, either $f\left(s_{t-2}\right) \geq f\left(s_{t-1}\right)$ or $f\left(s_{t}\right) \geq f\left(s_{t-1}\right)$, and we can reduce the size of $\operatorname{supp}(X) \cup \operatorname{supp}(Y)$ by 1 . However, this contradicts our induction hypothesis. 
The following corollary refutes the existence of a polar-pair of point-sets with large gap in any dimension. The proof follows from Theorem 2 and is given in the full version of the paper.

- Corollary 7 (No Polar-Pair of Point-Sets in $L^{1}$ with Large Gap). For any $\alpha>0$, there exist no subsets $A, B \subseteq \mathbb{R}^{d}$ of $n / 2$ vectors with $d<n / 2$ such that

- For any $u, v$ both in $A$, or both in $B,\|u-v\|_{1} \geq \frac{1}{1-2 / n} \cdot \alpha$.

- For any $u \in A$ and $v \in B,\|u-v\|_{1}<\alpha$.

We can show similar results that there are no polar-pairs of point-sets with large gap in the $L^{0}$ and $L^{2}$ metrics. The case of the $L^{0}$-metric follows directly from Theorem 2 when the alphabet set is $\{0,1\}$. (Please also see Lemma 9 for an alternate proof.) The case of the $L^{2}$-metric follows from the fact that $\operatorname{bsph}\left(L^{2}\right)=\Omega(n)[11,23]$ and that we can reduce the dimension of a polar-pairs of point-sets with constant gap to $O(\log n)$ using dimension reduction [17].

\section{Geometric representation of biclique in $L^{0}$}

In this section, we prove a lower bound on $\operatorname{bsph}\left(L^{0}\right)$ and an upper bound on bcd $\left(L^{0}\right)$. We start by providing a real-to-binary reduction below. Then we proceed to prove the lower bound on $\operatorname{bsph}\left(L^{0}\right)$ in Section 4.1 and then the upper bounds on $\operatorname{bcd}\left(L^{0}\right)$ in Section 4.2.

First we state the following (trivial) lemma, which allows mapping from vectors in $\mathbb{R}^{d}$ to zero-one vectors. The proof of the lemma can be found in the full version of the paper.

- Lemma 8 (Real to Binary Reduction). Let $S \subseteq \mathbb{R}$ be a finite set of real numbers. Then there exists a transformation $\phi: S^{d} \rightarrow\{0,1\}^{d|S|}$ such that, for any $x, y \in S^{d}$,

$$
\|x-y\|_{0}=\frac{1}{2} \cdot\|\phi(x)-\phi(y)\|_{0}
$$

\subsection{Lower bound on the biclique-sphericity}

Now we will show that $\operatorname{bsph}\left(L^{0}\right) \geq n$. Our proof requires the following lemma, which rules out a randomized algorithm that generates a polar-pair of point-sets.

- Lemma 9 (No Distribution for $L^{0}$ ). For any $\alpha>\beta \geq 0$, regardless of dimension, there exist no distributions $\mathcal{A}$ and $\mathcal{B}$ of points in $\mathbb{R}^{d}$ with finite supports such that

- $\mathbb{E}_{x, x^{\prime} \in_{R} \mathcal{A}}\left[\left\|x-x^{\prime}\right\|_{0}\right] \geq \alpha$.

- $\mathbb{E}_{y, y^{\prime} \in_{R} \mathcal{B}}\left[\left\|y-y^{\prime}\right\|_{0}\right] \geq \alpha$.

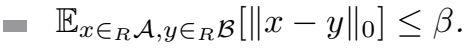

Proof. We prove by contradiction. Assume to a contrary that such distributions exist. Then

$$
\mathbb{E}_{x, x^{\prime} \in_{R} \mathcal{A}}\left[\left\|x-x^{\prime}\right\|_{0}\right]+\mathbb{E}_{y, y^{\prime} \in_{R} \mathcal{B}}\left[\left\|y-y^{\prime}\right\|_{0}\right]-2 \mathbb{E}_{x \in_{R} \mathcal{A}, y \in_{R} \mathcal{B}}\left[\|x-y\|_{0}\right]>0 .
$$

Let $A$ and $B$ be supports of $\mathcal{A}$ and $\mathcal{B}$, respectively. By Lemma 8, we may assume that vectors in $A$ and $B$ are binary vectors. Observe that each coordinate of vectors in $A$ and $B$ contribute to the expectations independently. In particular, Eq. (9) can be written as

$$
2 \sum_{i} \rho_{0, i}^{A} \rho_{1, i}^{A}+2 \sum_{i} \rho_{0, i}^{B} \rho_{1, i}^{B}+2 \sum_{i}\left(\rho_{0, i}^{A} \rho_{1, i}^{B}+\rho_{0, i}^{B} \rho_{1, i}^{A}\right)>0
$$

where $\rho_{0, i}^{A}, \rho_{1, i}^{A}, \rho_{0, i}^{B}$ and $\rho_{1, i}^{B}$ are the probability that the $i$-th coordinate of $x \in A$ (resp., $y \in B$ ) is 0 (resp., 1). Thus, to show a contradiction, it is sufficient to consider the coordinate 
which contributes the most to the summation in Eq. (10). The contribution of this coordinate to the summation is

$$
2 \rho_{0}^{A} \rho_{1}^{A}+2 \rho_{0}^{B} \rho_{1}^{B}-2\left(\rho_{0}^{A} \rho_{1}^{B}+\rho_{1}^{A} \rho_{0}^{B}\right)=2\left(\rho_{0}^{A}-\rho_{0}^{B}\right)\left(\rho_{1}^{A}-\rho_{1}^{B}\right)
$$

Since $\rho_{0}^{A}+\rho_{1}^{A}=1$ and $\rho_{0}^{B}+\rho_{1}^{B}=1$, the summation in Eq.(11) can be non-negative only if $\rho_{0}^{A}=\rho_{0}^{B}$ and $\rho_{1}^{A}=\rho_{1}^{B}$. But, then this implies that the summation in Eq.(11) is zero. We have a contradiction since this coordinate contributes the most to the summation in Eq. (10) which we assume to be positive.

The next Theorem shows that $\operatorname{bsph}\left(L^{0}\right) \geq n$.

- Theorem 10 (Lower Bound for $L^{0}$ with Arbitrary Alphabet). For any integers $\alpha>\beta \geq 0$ and $n>0$, there exist no subsets $A, B \subseteq \mathbb{R}^{d}$ of $n$ vectors with $d<n$ such that

- For any $a, a^{\prime} \in A,\left\|a-a^{\prime}\right\|_{0} \geq \alpha$.

- For any $b, b^{\prime} \in B,\left\|b-b^{\prime}\right\|_{0} \geq \alpha$.

- For any $a \in A$ and $b \in B,\|a-b\|_{0} \leq \beta$.

Proof. Suppose for a contradiction that such subsets $A$ and $B$ exist with $d<n$. We build uniform distributions $\mathcal{A}$ and $\mathcal{B}$ by uniformly at random picking a vector in $A$ and $B$, respectively. Then it is easy to see that the expected value of inner distance is

$$
\mathbb{E}_{x, x^{\prime} \in R} \mathcal{A}\left[\left\|x-x^{\prime}\right\|_{0}\right] \geq \alpha-\frac{\alpha}{n}
$$

The intra distance of $B$ is similar. We know that $\alpha-\beta \geq 1$ because they are integers and so are $L^{0}$-distances. But, then if $\alpha<n$, we would have distributions that contradict Lemma 9 . Note that $\alpha$ and $\beta$ are at most $d$ (dimension). Therefore, we conclude that $d \geq n$.

\subsection{Upper bound on the biclique contact-dimension}

Now we show that $\operatorname{bcd}\left(L^{0}\right) \leq n$.

- Theorem 11 (Upper Bound for $L^{0}$ with Arbitrary Alphabet). For any integer $n>0$ and $d=n$, there exist subsets $A, B \subseteq \mathbb{R}^{d}$ each with $n$ vectors such that

- For any $a, a^{\prime} \in A,\left\|a-a^{\prime}\right\|_{0}=d$.

- For any $b, b^{\prime} \in B,\left\|b-b^{\prime}\right\|_{0}=d$.

- For any $a \in A$ and $b \in B,\|a-b\|_{0}=d-1$.

Proof. First we construct a set of vectors $A$. For $i=1,2, \ldots, n$, we define the $i$-th vector $a$ of $A$ so that $a$ is an all- $i$ vector. That is, $a=(i, i, \ldots, i)$. Next we construct a set of vectors $B$. The first vector of $B$ is $(1,2, \ldots, n)$. Then the $(i+1)$-th vector of $B$ is the left rotation of the $i$-th vector. Thus, the $i$-th vector of $B$ is $b=(i, i+1, \ldots, n, 1,2, \ldots, i-1)$.

It can be seen that the $L^{0}$-distance between any two vectors from the same set is $d$ because all the coordinates are different. Any vectors from different set, say $a \in A$ and $b \in B$, must have at least one common coordinate. Thus, their $L^{0}$-distance is $d-1$. This proves the lemma.

Below is the upper bound for zero-one vectors, which is a corollary of Theorem 11 . The proof can be found in the full version of the paper.

Corollary 12 (Upper Bound for $L^{0}$ with Binary Vectors). For any integer $n>0$ and $d=n^{2}$, there exist subsets $A, B \subseteq \mathbb{R}^{d}$ each with $n$ vectors such that

- For any $a, a^{\prime} \in A,\left\|a-a^{\prime}\right\|_{0}=n$.

- For any $b, b^{\prime} \in B,\left\|b-b^{\prime}\right\|_{0}=n$.

- For any $a \in A$ and $b \in B,\|a-b\|_{0}=n-1$. 


\section{Geometric representation of biclique in $L^{p}$ for $p \in(1,2)$}

In this section, we prove the upper bound on $\operatorname{bcd}\left(L^{p}\right)$ for $p \in(1,2)$. We are unable to show any lower bound for these $L_{p}$-metrics except for the lower bound of $\Omega(\log n)$ obtained from the $\epsilon$-net lower bound in Theorem 16 (which will be proven in the next Section).

- Theorem 13 (Upper Bound for $L^{p}$ with $1<p<2$ ). For every $1<p<2$ and for all integers $n \geq 1$, there exist two sets $A, B \subseteq \mathbb{R}^{2 n}$ each of cardinality $n$ such that the following holds:

1. For every distinct points $u, v \in A,\|u-v\|_{p}=2^{1 / p}$.

2. For every distinct points $u, v \in B,\|u-v\|_{p}=2^{1 / p}$.

3. For every points $u \in A$ and $v \in B,\|u-v\|_{p}<2^{1 / p}$.

Proof. We will construct point-sets as claimed in the theorem for given $p$ and $n$. Let $\alpha$ be a parameter depending on $p$ and $n$, which will be set later. For each $i \in[n]$, we create a point $a \in A$ by setting

$$
a_{j}= \begin{cases}0 & \text { if } 1 \leq j \leq n \text { and } j \neq i \\ 1 & \text { if } 1 \leq j \leq n \text { and } i=j \\ \alpha & \text { if } n+1 \leq j \leq 2 n\end{cases}
$$

Similarly, for each $i \in[n]$, we create a point $b \in B$ by setting

$$
b_{j}= \begin{cases}\alpha & \text { if } 1 \leq j \leq n \\ 0 & \text { if } n+1 \leq j \leq 2 n \text { and } j \neq n+i \\ 1 & \text { if } n+1 \leq j \leq 2 n \text { and } j=n+i\end{cases}
$$

By construction, for every pair of points $u, v$ both in $A$ or both in $B$, their $L^{p}$-distance is $\|u-v\|_{p}=2^{1 / p}$, and for every pair of points from different sets, say $u \in A$ and $v \in B$, their $L^{p}$-distance is

$$
\|u-v\|_{p}=2^{1 / p} \cdot\left((1-\alpha)^{p}+(n-1) \cdot \alpha^{p}\right)^{1 / p} \leq 2^{1 / p} \cdot\left((1-\alpha)^{p}+n \cdot \alpha^{p}\right)^{1 / p}
$$

Now let us choose $\alpha>n^{-1 /(p-1)}$, and consider the term $(1-\alpha)^{p}+n \cdot \alpha^{p}$ in Eq. (12). Observe that $\alpha<n \cdot \alpha^{p}$ for $1<p<2$. Define a function $f(x)=(1-\alpha)^{x}+n \cdot \alpha^{x}$. We know that $f(x)$ is less than 1 as $x$ goes from $\infty$ to 1 (i.e., $\lim _{x \rightarrow 1^{+}}\left((1-\alpha)^{x}+n \cdot \alpha^{x}\right)<1$ ). Moreover, $f(x)$ is decreasing for $0<\alpha<1$, which means that $f(p)<1$. Consequently, $\|u-v\|_{p}<2^{1 / p}$, and the theorem follows.

To finish the proof, we will show that $f(x)$ is decreasing for $x>1$ provided that $0<\alpha<1$. It suffices to show that $f^{\prime}(x)<0$ for all values of $x$.

$$
f^{\prime}(x)=\frac{\partial}{\partial x}\left((1-\alpha)^{x}+n \cdot \alpha^{x}\right)=(1-\alpha)^{x} \ln (1-\alpha)+n \cdot \alpha^{x} \ln (\alpha)<0 .
$$

The last inequality follows from the fact $\ln (x)<0$ for $0<x<1$ and that $0<\alpha, 1-\alpha<1$.

\section{Geometric representation of biclique in $L^{p}$ for $p>2$}

In this section, we show the lower bound on $\operatorname{bsph}\left(L^{p}\right)$ and an upper bound on $\operatorname{bcd}\left(L^{p}\right)$ for $p>2$. Both bounds are logarithmic. The latter upper bound is constructive and efficient (in the sense that the polar-pair of point-sets can be constructed in $\widetilde{O}(n)$-time). This implies the subquadratic-time equivalence between Closest PAIR and BCP. 


\subsection{Lower bound on the biclique sphericity}

Now we show the lower bound on the biclique sphericity of a complete bipartite graph in $L^{p}$-metrics with $p>2$. In fact, we prove the lower bound for the case of a star graph on $n$ vertices, denoted by $S_{n}$, and then use the fact that $\operatorname{bsph}(H) \leq \operatorname{bsph}(G)$ for all induced subgraph $H$ of $G$ (i.e., $\left.\operatorname{bsph}\left(K_{n / 2, n / 2}, L^{p}\right) \geq \operatorname{bsph}\left(S_{n / 2}, L^{p}\right)\right)$.

In short, we show in Lemma 16 that $O(\log n)$ is the maximum number of $L^{p}$-balls of radius $1 / 2$ that we can pack in an $L^{p}$-ball of radius one so that no two of them intersect or touch each other. This upper bounds, in turn, implies the lower bound on the dimension. We proceed with the proof by volume arguments, which are commonly used in proving the minimum number of points in an $\epsilon$-net that are sufficient to cover all the points in a sphere.

- Definition 14 ( $\epsilon$-net). The unit $L^{p}$-ball in $\mathbb{R}^{d}$ centered at $o$ is denoted by

$$
\mathbb{B}\left(L_{p}^{d}, o\right)=\left\{x \in \mathbb{R}^{d} \mid\|x-o\|_{p} \leq 1\right\} .
$$

For brevity, we write $\mathbb{B}\left(L_{p}^{d}\right)$ to mean $\mathbb{B}\left(L_{p}^{d}, o\right)$. Let $(X, d)$ be a metric space and let $S$ be a subset of $X$ and $\epsilon$ be a constant greater than 0 . A subset $N_{\epsilon}$ of $X$ is called an $\epsilon$-net of $S$ under $d$ if for every point $x \in S$ it holds for some point $y \in N_{\epsilon}$ that $d(x, y) \leq \epsilon$.

The following lemma is well known in literature (see, e.g., [28]). For the sake of completeness, we provide a proof in the full version of the paper.

- Lemma 15. There exists an $\epsilon$-net for $\mathbb{B}\left(L_{p}^{d}\right)$ under the $L^{p}$-metric of cardinality $\left(1+\frac{2}{\epsilon}\right)^{d}$.

- Theorem 16. For every $N, d \in \mathbb{N}$, for $p \geq 1$, and for any two sets $A, B \subseteq \mathbb{R}^{d}$, each of cardinality $N$, suppose the following holds for some non-negative real numbers $\alpha$ and $\beta$ with $\alpha>\beta$.

1. For every $u$ and $v$ both in $A,\|u-v\|_{p}>\alpha$.

2. For every $u$ and $v$ both in $B,\|u-v\|_{p}>\alpha$.

3. For every $u$ in $A$ and $v$ in $B,\|u-v\|_{p} \leq \beta$.

Then the dimension $d$ must be at least $\log _{5}(N)$.

Proof. Scale and translate the sets $A, B$ in such a way that $\beta=1$ and that $\overrightarrow{0} \in B$. It follows that $A \subseteq \mathbb{B}\left(L_{p}^{d}\right)$. By Lemma 15 , we can fix a $1 / 2$-net $N_{1 / 2}$ for $\mathbb{B}\left(L_{p}^{d}\right)$ of size $5^{d}$. Note that, for every $x \in N_{1 / 2}$, the ball $1 / 2 \cdot \mathbb{B}\left(L_{p}^{d}, x\right)$ contains at most one point from $A$. Note also that $N_{1 / 2}$ covers $\mathbb{B}\left(L_{p}^{d}\right)$. Thus, $|A| \leq 5^{d}$ which implies that $d \geq \log _{5}(N)$.

\subsection{Upper bound on the biclique contact-dimension}

We first give a simple randomized construction that gives a logarithmic upper bound on the biclique contact-dimension of $L^{p}$. The construction is simple. We uniformly at random take a subset $A$ of $n$ vectors from $\{-1,1\}^{d / 2} \times\{0\}^{d / 2}$ and a subset $B$ of $n$ vectors from $\{0\}^{d / 2} \times\{-1,1\}^{d / 2}$. Observe that, for any $p>2$, the $L^{p}$-distance of any pair of vectors $u \in A$ and $v \in B$ is exactly $d$ while the expected distance between the inner pair $u, u^{\prime} \in A$ (resp., $v, v^{\prime} \in B$ ) is strictly larger than $d$. Thus, if we choose $d$ to be sufficiently large, e.g., $d \geq 10 \ln n$, then we can show by a standard concentration bound (e.g., Chernoff's bound) that the probability that the inner-pair distance is strictly larger than $d$ is at least $1-1 / n^{3}$. Applying the union bound over all inner-pairs, we have that the $d$-neighborhood graph of $A \cup B$ is a bipartite complete graph with high probability. Moreover, the distances between any crossing pairs $u \in A$ and $v \in B$ are the same for all pairs. This shows the upper bound for the contact-dimension of a biclique in the $L^{p}$-metric for $p>2$. 
The above gives a simple proof of the upper bound on the biclique contact-dimension of the $L^{p}$-metric. Moreover, it shows a randomized construction of the polar-pair in the $O(\log n)$-dimensional $L^{p}$-metric, for $p>2$, thus implying that Closest PAIR and BCP are equivalent for these $L^{p}$-metrics.

For algorithmic purposes, we provide a deterministic construction below using appropriate binary codes.

- Theorem 17. For any $p>2$, let $\zeta=2^{p-3}$. There exist two sets $|A|=|B|=n$ of vectors in $\mathbb{R}^{d}$, where $d=2 \alpha \log _{2} n$, for some constant $\alpha \geq 1$, such that the following holds.

1. For all $u, u^{\prime} \in A,\left\|u-u^{\prime}\right\|_{p}>((\zeta+1 / 2) d)^{1 / p}$.

2. For all $v, v^{\prime} \in B,\left\|v-v^{\prime}\right\|_{p}>((\zeta+1 / 2) d)^{1 / p}$.

3. For all $u \in A, v \in B,\|u-v\|_{p}=d^{1 / p}$.

Moreover, there exists a deterministic algorithm that outputs $A$ and $B$ in time $\widetilde{O}(n)$.

Proof. In literature, we note that for any constant $\delta>0$, there is an explicit binary code of (some) constant relative rate and relative distance at least $\frac{1}{2}-\delta$ and the entire code can be listed in quasilinear time with respect to the size of the code (see Appendix E.1.2.5 from [13], or Justesen codes [18]). To be more specific, we can construct in $O(n \log O(1) n)$-time a set $C \subseteq\{-1,1\}^{d^{\prime}}$ such that (1) $|C|=n,(2) d^{\prime}=d / 2=\alpha \log _{2} n$ for some constant $\alpha \geq 1$ and (3) for every two vectors $x, y \in C, x$ and $y$ differ on at least $\left(\frac{1}{2}-\delta\right) d^{\prime}$ coordinates, for some constant $\delta \in\left(0, \frac{1}{4}-\frac{1}{2^{p}}\right)$.

We construct the sets $A$ and $B$ as subsets of $\{-1,0,1\}^{d}$. For every $i \in[n]$, the $i^{\text {th }}$ point of $A$ is given by the concatenation of the $i^{\text {th }}$ point of $C$ with $0^{d^{\prime}}$. Similarly, the $i^{\text {th }}$ point of $B$ is given by the concatenation of $0^{d^{\prime}}$ with the $i^{\text {th }}$ point of $C$ (note the reversal in the order of the concatenation). In particular, points in $A$ and $B$ are of the form $\left(x_{i}, \overrightarrow{0}\right)$ and $\left(\overrightarrow{0}, x_{i}\right)$, respectively, where $x_{i}$ is the $i^{\text {th }}$ point in $C$ and $\overrightarrow{0}$ is the zero-vector of length $\alpha \log _{2} n$.

First, consider any two points in the same set, say $u, u^{\prime} \in A$ (resp., $v, v^{\prime} \in B$ ). We have from the distance of $C$ that on at least $\left(\frac{1}{2}-\delta\right) d^{\prime}$ coordinates the two points differ by 2 , thus implying that their $L^{p}$-distance is at least

$$
\left(\left(\frac{1}{2}-\delta\right) d^{\prime} 2^{p}\right)^{1 / p}>\left(\left(\frac{1}{4}+\frac{1}{2^{p}}\right) d^{\prime} 2^{p}\right)^{1 / p}=\left(\left(2^{p-3}+\frac{1}{2}\right) d\right)^{1 / p}
$$

This proves the first two items of the theorem. Next we prove the third item. Consider any two points from different sets, say $u \in A$ and $v \in B$. It is easy to see from the construction that $u$ and $v$ differ in every coordinate by exactly 1 . Thus, the $L^{p}$-distance between any two points from different set is exactly

$$
\left(2 d^{\prime}\right)^{1 / p}=d^{1 / p}
$$

\section{Fine-grained complexity of CLOSEST PAIR in $L^{\infty}$}

In this section, we prove the quadratic-time hardness of CLOSEST PAIR in the $L^{\infty}$-metric. Our reduction is from the Orthogonal Vectors problem (OV), which we phrase it as follows. Given a pair of collections of vectors $U, W \subseteq\{0,1\}^{d}$, the goal is to find a pair of vectors $u \in U$ and $w \in W$ such that $\left(u_{i}, w_{i}\right) \in\{(0,0),(0,1),(1,0)\}$ for all $i \in[d]$. Throughout, we denote by $n$ the total number of vectors in $U$ and $W$.

Let $U, W \subseteq\{0,1\}^{d}$ be an instance of OV. We may assume that $U$ and $W$ have no duplicates. Otherwise, we may sort vectors in $U$ (resp., $W$ ) in lexicographic order and then sequentially remove duplicates; this preprocessing takes $O(d n \log n)$-time. 
We construct a pair of sets $A, B \subseteq \mathbb{R}^{d}$ of BCP from $U, W$ as follows. For each vector $u \in U$ (resp., $w \in W$ ), we create a point $a \in A$ (resp., $b \in B$ ) such that

$$
\begin{gathered}
a_{j}= \begin{cases}0 & \text { if } u_{j}=0 \\
2 & \text { if } u_{j}=1\end{cases} \\
b_{j}= \begin{cases}1 & \text { if } w_{j}=0 \\
-1 & \text { if } w_{j}=1\end{cases}
\end{gathered}
$$

Observe that, for any vectors $a \in A$ and $b \in B,\left|a_{j}-b_{j}\right|=3$ only if $u_{j}=w_{j}=1$; otherwise, $\left|a_{j}-b_{j}\right|=1$. It can be seen that $\|a-b\|_{p}=d$ if and only if their corresponding vectors $u \in U$ and $w \in W$ are orthogonal. Thus, this gives an alternate proof for the quadratic-time hardness of BCP under $\mathrm{OVH}$.

Here we show that the reduction above rules out both exact and $(2-o(1))$-approximation algorithm for Closest PAIR in $L^{\infty}$ that runs in subquadratic-time (unless OVH is false). That is, we prove Theorem 5, which follows from the theorem below whose proof is in the full version of the paper. In short, given an instance $(U, W)$ of the OV problem, the instance of Closest PAIR that is constructed in the reduction in simply $A \cup B$.

Theorem 18. Assuming $O V H$, for any $\varepsilon>0$ and $d=\omega(\log n)$, there is no $O\left(n^{2-\varepsilon}\right)$-time algorithm that, given a point-set $P \subseteq \mathbb{R}^{d}$, distinguishes between the following two cases:

- There exists a pair of vectors in $P$ with $L^{\infty}$-distance one.

- Every pair of vectors in $P$ has $L^{\infty}$-distance two.

In particular, approximating CLOSEST PAIR in the $L^{\infty}$-metric to within a factor of two is at least as hard as solving the Orthogonal Vectors problem.

\section{Conclusion and discussion}

We have studied the sphericity and contact dimension of the complete bipartite graph in various metrics. We have proved lower and upper bounds on these measures for some metrics. However, biclique sphericity and biclique contact dimension in the $L^{1}$-metric remains poorly understood as we are unable to show any strong upper or lower bounds. However, we believe that both $L^{1}$ and $L^{2}$ metrics have linear upper and lower bounds. To be precise, we raise the following conjecture:

- Conjecture 19 ( $L^{1}$-Biclique Sphericity Conjecture).

$$
\operatorname{bsph}\left(L^{1}\right)=\Omega(n)
$$

We have also shown conditional lower bounds for the Closest Pair problem in the $L^{p}$ metric, for all $p \in \mathbb{R}_{>2} \cup\{\infty\}$, by using polar-pair of point-sets. However, it is unlikely that our techniques could get to the regime of $L^{2}, L^{1}$, and $L^{0}$, which are popular metrics. An open question is thus whether there exists an alternative technique to derive a lower bound from $\mathrm{OVH}$ to the Closest Pair problem for these metrics. The answer might be on the positive side, i.e., there might exist an algorithm that performs well in the $L^{2}$-metric because there are more tools available, e.g., Johnson-Lindenstrauss' dimension reduction. Thus, it is possible that there exists a strongly subquadratic-time algorithm in the $L^{2}$-metric. This question remains an outstanding open problem. 


\section{References}

1 Amir Abboud, Aviad Rubinstein, and R. Ryan Williams. Distributed PCP theorems for hardness of approximation in P. In 58th IEEE Annual Symposium on Foundations of Computer Science, FOCS 2017, Berkeley, CA, USA, October 15-17, 2017, pages 25-36, 2017. doi:10.1109/FOCS. 2017. 12.

2 Amir Abboud, Aviad Rubinstein, and Ryan Williams. Distributed PCP theorems for hardness of approximation in P. CoRR, abs/1706.06407, 2017. Preliminary version in FOCS'17. URL: http://arxiv.org/abs/1706.06407, arXiv:1706.06407.

3 Josh Alman and Ryan Williams. Probabilistic polynomials and hamming nearest neighbors. In IEEE 56th Annual Symposium on Foundations of Computer Science, FOCS 2015, Berkeley, CA, USA, 17-20 October, 2015, pages 136-150, 2015. doi:10.1109/FOCS.2015.18.

4 Noga Alon and Pavel Pudlák. Equilateral sets in $\ell_{p}^{n}$. Geometric 6 Functional Analysis GAFA, 13(3):467-482, 2003. doi:10.1007/s00039-003-0418-7.

5 Hans-Jürgen Bandelt, Victor Chepoi, and Monique Laurent. Embedding into rectilinear spaces. Discrete \& Computational Geometry, 19(4):595-604, 1998. doi:10.1007/ PL00009370.

6 Michael Ben-Or. Lower bounds for algebraic computation trees (preliminary report). In Proceedings of the 15th Annual ACM Symposium on Theory of Computing, 25-27 April, 1983, Boston, Massachusetts, USA, pages 80-86, 1983. doi:10.1145/800061.808735.

7 Jon Louis Bentley. Multidimensional divide-and-conquer. Commun. ACM, 23(4):214-229, 1980. doi:10.1145/358841.358850.

8 Jon Louis Bentley and Michael Ian Shamos. Divide-and-conquer in multidimensional space. In Proceedings of the 8th Annual ACM Symposium on Theory of Computing, May 3-5, 1976, Hershey, Pennsylvania, USA, pages 220-230, 1976. doi:10.1145/800113.803652.

9 Yonatan Bilu and Nathan Linial. Monotone maps, sphericity and bounded second eigenvalue. J. Comb. Theory, Ser. B, 95(2):283-299, 2005. doi:10.1016/j.jctb.2005.04.005.

10 Michel Deza and Hiroshi Maehara. A few applications of negative- type inequalities. Graphs and Combinatorics, 10(2-4):255-262, 1994. doi:10.1007/BF02986674.

11 Peter Frankl and Hiroshi Maehara. On the contact dimensions of graphs. Discrete $\&$ Computational Geometry, 3:89-96, 1988. doi:10.1007/BF02187899.

12 Omer Gold and Micha Sharir. Dominance products and faster algorithms for highdimensional closest pair under \$1_\infty\$. CoRR, abs/1605.08107, 2016. URL: http: //arxiv.org/abs/1605.08107, arXiv:1605.08107.

13 Oded Goldreich. Computational Complexity: A Conceptual Perspective. Cambridge University Press, New York, NY, USA, 1 edition, 2008.

14 Richard K Guy. An olla-podrida of open problems, often oddly posed. The American Mathematical Monthly, 90(3):196-200, 1983.

15 Klaus H. Hinrichs, Jürg Nievergelt, and Peter Schorn. Plane-sweep solves the closest pair problem elegantly. Inf. Process. Lett., 26(5):255-261, 1988. doi:10.1016/0020-0190(88) 90150-0.

16 Piotr Indyk, Moshe Lewenstein, Ohad Lipsky, and Ely Porat. Closest pair problems in very high dimensions. In Automata, Languages and Programming: 31st International Colloquium, ICALP 2004, Turku, Finland, July 12-16, 2004. Proceedings, pages 782-792, 2004. doi : 10.1007/978-3-540-27836-8_66.

17 William B Johnson and Joram Lindenstrauss. Extensions of lipschitz mappings into a hilbert space. Contemporary mathematics, 26(189-206):1, 1984.

18 Jørn Justesen. Class of constructive asymptotically good algebraic codes. IEEE Trans. Information Theory, 18(5):652-656, 1972. doi:10.1109/TIT.1972.1054893.

19 Samir Khuller and Yossi Matias. A simple randomized sieve algorithm for the closest-pair problem. Inf. Comput., 118(1):34-37, 1995. doi:10.1006/inco.1995.1049. 
20 Jack H. Koolen, Monique Laurent, and Alexander Schrijver. Equilateral dimension of the rectilinear space. Des. Codes Cryptography, 21(1/3):149-164, 2000.

21 Hiroshi Maehara. Space graphs and sphericity. Discrete Applied Mathematics, 7(1):55-64, 1984.

22 Hiroshi Maehara. Contact patterns of equal nonoverlapping spheres. Graphs and Combinatorics, 1(1):271-282, 1985. doi:10.1007/BF02582952.

23 Hiroshi Maehara. Dispersed points and geometric embedding of complete bipartite graphs. Discrete \& Computational Geometry, 6:57-67, 1991. doi:10.1007/BF02574674.

24 Michael O. Rabin. Probabilistic algorithms. In Proceedings of a Symposium on New Directions and Recent Results in Algorithms and Complexity, Computer Science Department, Carnegie-Mellon University, April 7-9, 1976, pages 21-39, 1976.

25 Fred S Roberts. On the boxicity and cubicity of a graph. Recent Progresses in Combinatorics, pages 301-310, 1969.

26 Aviad Rubinstein. Hardness of approximate nearest neighbor search. CoRR, abs/1803.00904, 2018. arXiv:1803.00904.

27 Michael Ian Shamos and Dan Hoey. Closest-point problems. In 16th Annual Symposium on Foundations of Computer Science, Berkeley, California, USA, October 13-15, 1975, pages 151-162, 1975. doi:10.1109/SFCS.1975.8.

28 Roman Vershynin. Introduction to the non-asymptotic analysis of random matrices. CoRR, abs/1011.3027, 2010. URL: http://arxiv.org/abs/1011.3027, arXiv:1011.3027.

29 Ryan Williams. A new algorithm for optimal 2-constraint satisfaction and its implications. Theor. Comput. Sci., 348(2-3):357-365, 2005. Preliminary version in ICALP'04. doi: $10.1016 / j \cdot$ tcs. 2005.09 .023 .

30 Ryan Williams. On the difference between closest, furthest, and orthogonal pairs: Nearlylinear vs barely-subquadratic complexity in computational geometry. arXiv preprint arXiv:1709.05282, 2017.

31 Virginia Vassilevska Williams. Hardness of easy problems: Basing hardness on popular conjectures such as the strong exponential time hypothesis (invited talk). In 10th International Symposium on Parameterized and Exact Computation, IPEC 2015, September 16-18, 2015, Patras, Greece, pages 17-29, 2015. doi:10.4230/LIPIcs.IPEC.2015.17.

32 Virginia Vassilevska Williams. Fine-grained algorithms and complexity (invited talk). In 33rd Symposium on Theoretical Aspects of Computer Science, STACS 2016, February 17-20, 2016, Orléans, France, pages 3:1-3:1, 2016. doi:10.4230/LIPIcs.STACS.2016.3.

33 Andrew Chi-Chih Yao. Lower bounds for algebraic computation trees with integer inputs. SIAM J. Comput., 20(4):655-668, 1991. Preliminary version in FOCS'89. doi:10.1137/ 0220041. 